\title{
Effect of Weed Management Practices on Weed Density, Dry Weight and Growth of Kodo Millet (Paspalum scrobiculatum L.)
}

\author{
G. K. Meghana, T. S. Sukanya, R. M. Salmankhan* and H. P. Kiran \\ Department of Agronomy, College of Agriculture, UAS, GKVK, Bangalore, India \\ *Corresponding author
}

\section{A B S T R A C T}

\section{Keywords}

Kodo millet, Weed density, Weed dry weight, Phytotoxicity and Weed control efficiency

\section{Article Info}

Accepted: 24x October 2020 Available Online: 10 November 2020
An experiment in Kodo millet was conducted during Kharif 2018 in red sandy loamy soil of Zonal Agricultural Research Station, University of Agricultural Sciences, GKVK, Bengaluru with different weed management practices. Twelve treatments replicated thrice in a randomized complete block design (RCBD). The weed density (4.59, 4.67 and 4.69 $\left.\mathrm{m}^{-2}\right)$ and weed dry weight $\left(9.00,7.00\right.$ and $\left.3.71 \mathrm{~g} \mathrm{~m}^{-2}\right)$ were found significantly lower at 30 , 45 days after sowing and at harvest, respectively in $\mathrm{T}_{5}$ i.e. the application of Bensulfuron methyl $0.6 \% \mathrm{G}+$ Pretilachlor $6.0 \% \mathrm{G} 165 \mathrm{~g}$ a. i. ha $^{-1}$ as pre emergent herbicide $f b$ Bispyribac sodium $10 \%$ SC $10 \mathrm{~g}$ a. i. ha ${ }^{-1}$ among herbicide treatments was found supreme in controlling weeds growth and thereby enhancing the performance of crop and this treatment was found on found par with the treatment i.e. Bensulfuron methyl $0.6 \% \mathrm{G}+$ Pretilachlor $6.0 \%$ G $165 \mathrm{~g}$ a. i. ha $^{-1} \mathrm{fb}$ Ethoxysulfuron $15 \%$ WG $12 \mathrm{~g}$ a. i. ha $^{-1}(4.38,4.78$ and $4.72 \mathrm{~m}^{-2}$ of weed density and $7.33,7.50$ and $8.27 \mathrm{~g} \mathrm{~m}^{-2}$ of weed dry weight), two intercultivation followed by hand weeding at 20 and 40 DAS $\left(3.40,3.17\right.$ and $3.78 \mathrm{~m}^{-2}$, and $4.00,2.63$ and $2.32 \mathrm{gm}^{-2}$ at $30,45 \mathrm{DAS}$ and at harvest, respectively) indicating the excelled efficiency of Bensulfuron methyl + pretilachlor $f b$ Bispyribacsodium in controlling sedges, grasses and broad leaf weeds throughout the growth stages of kodo millet without any phytotoxicity on kodo millet crop.

\section{Introduction}

Kodo millet (Paspalum scrobiculatum L.) popularly known as Haraka in Karnataka is one of the important nutri cereals. It is a coarsest millet crop which is having a high drought tolerance capacity due to its deep root system and nutritionally superior over rice and wheat in terms of higher protein, dietary fibre and lesser fat content, which play an important role in the energy requirement and nutrient intake of human. It is also having a good fodder value, has immense potentiality and hence found the importance in rainfed areas of Madhya Pradesh, Chhattisgarh, Maharashtra, Tamil Nadu, Bihar and Karnataka.

It is grown as a minor crop in many Asian countries, with the exception of the Deccan plateau in India where it is grown as a food source. It grows wild as a perennial in the west of Africa, where it is eaten as a famine food. Kodo millet is mainly cultivated for 
human consumption in Philippines, Indonesia, Vietnam, Thailand, West Africa and India. Nutri cereals are grown worldwide and in India, it is cultivated over an area of $0.62 \mathrm{~m}$. ha with the production of $0.44 \mathrm{~m}$. $t$. and with a productivity of $714 \mathrm{~kg} \mathrm{ha}{ }^{-1}$. Whereas, in Karnataka, nutria cereals occupy an area of 21,000 ha, with a production of $7000 \mathrm{t}$. production and with a productivity of $333 \mathrm{~kg}$ ha $^{-1}$.

Weeds are serious problem in Kodo millet, both in irrigated areas and assured rainfed situations during monsoon season where there is adequate moisture throughout the crop growth period. Due to slower initial growth, kodo millet is susceptible to weed competition. Weeds compete with crop plants for nutrients, moisture, space and light and reduce not only the crop yields but also quality of produce accounting a loss of about 37 per cent in agricultural produce depending upon factors like type of weed flora and its density in standing crop in India (Yaduraju, 2006). So, timely weed management is essential for achieving higher yield. In kodo millet weeds must be controlled during the initial 2-6 weeks period after which, the canopy develops thick enough to smother the weeds.

Conventionally, weeds are controlled by hoeing and animal drawn implements. But scarcity of human labour along with higher wages have made the weed management difficult to go for hand weeding an impossible task now a days.

The best alternate solution for weed management in such situations is use of herbicides along with other weed management practices. In recent years the usage of herbicides has revolutionized weed management practices in all crops particularly cereals in specific to nutri cereals in India and the use of herbicides in integrated weed management practices for effective weed management in kodo is quite effective to manage the composite weed flora.

\section{Materials and Methods}

The experiment to study the performance of different weedicides on yield and economics in kodo millet consisted of twelve treatments replicated thrice in a randomized complete block design (RCBD). This experiment was conducted during Kharif 2018 at Gandhi Krishi Vignan Kendra (GKVK). The soil is red sandy loam and the treatments tested were, $\mathrm{T}_{1}$ : Oxadiargyl $80 \%$ WP @150 g a. i. ha $^{-1}, \mathrm{~T}_{2}$ : Bensulfuron methyl $0.6 \% \mathrm{G}+$ Pretilachlor 6.0\% G @ 165 g a. i ha ${ }^{-1}, \mathrm{~T}_{3}$ : Pendimethalin $38.7 \%$ CS @ 450 g a. i ha ${ }^{-1}$, T4: Oxadiargyl $80 \%$ WP @ $150 \mathrm{~g} \mathrm{a.i} \mathrm{ha}{ }^{-1} f b$ Bispyribacsodium $10 \%$ SC @ 10 g a. i ha ${ }^{-1}$, $\mathrm{T}_{5}$ : Bensulfuron methyl $0.6 \% \mathrm{G}+$ Pretilachlor $6.0 \% \mathrm{G} @ 165 \mathrm{~g}$ a. i ha ${ }^{-1} \mathrm{fb}$ Bispyribacsodium 10\% SC @ $10 \mathrm{~g} \mathrm{a.} \mathrm{i} \mathrm{ha}{ }^{-1}$, $\mathrm{T}_{6}$ : Pendimethalin $38.7 \% \mathrm{CS} @ 450 \mathrm{~g}$ a. i ha ${ }^{1} \mathrm{fb}$ Bispyribac sodium $10 \%$ SC @10 g a. i ha $^{-1}, \mathrm{~T}_{7}$ : Oxadiargyl $80 \%$ WP @ 150 g a.i $\mathrm{ha}^{-1} \mathrm{fb}$ Ethoxysulfuron $15 \%$ WG @ $12 \mathrm{~g}$ a. i $\mathrm{ha}^{-1}, \mathrm{~T}_{8}$ : Bensulfuron methyl $0.6 \% \mathrm{G}+$ Pretilachlor 6.0\% G @ 165 g a. i ha ${ }^{-1} f b$

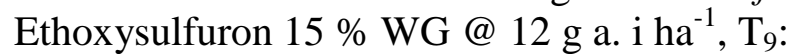
Pendimethalin 38.7\% CS@450 g a. i ha ${ }^{-1} \mathrm{fb}$ Ethoxysulfuron 15\%WG@12 g a. i ha ${ }^{-1}$, $\mathrm{T}_{10}$ : Two intercultivation + hand weeding @ 20 and 40 DAS, $\mathrm{T}_{11}$ : un weeded check and $\mathrm{T}_{12}$ : weed free check.

Kodo millet variety used was 'RBK-115' which was sown with a spacing of $30 \mathrm{~cm} \mathrm{x}$ $10 \mathrm{~cm}$. Observations on weed density, weed dry matter and other parameters were recorded in an area of $1 \mathrm{~m}^{2}$.

\section{Results and Discussion}

The results of the investigation revealed that at 30, 45 DAS and at harvest, significantly 
lower density and dry weight of sedge was observed in the treatment $\mathrm{T}_{4}$ i.e Oxadiargyl 80 \% WP 150 g a. i. ha ${ }^{-1} f b$ Bispyribac sodium $10 \%$ SC 10 g a. i. ha ${ }^{-1}$. Significantly lower number of grassy weeds was recorded with $\mathrm{T}_{5}$ i.e., Bensulfuron methyl $0.6 \%$ G + Pretilachlor $6.0 \%$ G 165 g a. i. ha $f b$ Bispyribac sodium $10 \%$ SC $10 \mathrm{~g}$ a. i. ha ${ }^{-1}$, however it was at par with $\mathrm{T}_{8}, \mathrm{~T}_{6}$ and $\mathrm{T}_{10}$ and rest of the treatments showed significantly higher incidence of weeds along with its dry weight.

The broad leaf weeds density and dry weight was significantly lower in $\mathrm{T}_{5}$ i.e., Bensulfuron methyl $0.6 \% \mathrm{G}+$ Pretilachlor $6.0 \% \mathrm{G} 165 \mathrm{~g}$ a. i. $\mathrm{ha}^{-1} \mathrm{fb}$ Bispyribac sodium $10 \%$ SC $10 \mathrm{~g}$ a. i. ha ${ }^{-1}$ at 20 DAS compared to rest of the other treatments except $T_{6}$ and $T_{10}$ in other they were found at par. During $45 \mathrm{DAS}$ and at harvest, Bensulfuron methyl $0.6 \% \mathrm{G}+$ Pretilachlor $6.0 \%$ G 165 g a. i. ha $^{1} f b$ Bispyribac sodium $10 \%$ SC $10 \mathrm{~g}$ a. i. ha ${ }^{-1}$ at 20 DAS $\left(T_{5}\right)$ recorded significantly lower total weed density and weed dry weight compared to other treatments and these findings are in line with the findings of Pradhan et al., (2010).

Among all the treatments, weed free check $\left(T_{12}\right)$ recorded the lowest sedge, grass, broad leaf weeds and total weed density as well as dry weight due to the physical uprooting of both above and below ground parts of weeds by the manual hand weeding imposed on during 20 and 40 DAS. Whereas, un-weeded control $\left(T_{11}\right)$ recorded the highest sedge, grass, broad leaf, total weed density and weed dry weight due to the non-interruption for growth of weeds.

Among herbicide treatments, Bensulfuron methyl $0.6 \% \mathrm{G}+$ Pretilachlor $6.0 \% \mathrm{G} 165 \mathrm{~g}$ a. i. $\mathrm{ha}^{-1} \mathrm{fb}$ Bispyribac sodium $10 \%$ SC $10 \mathrm{~g}$ a. i. ha $^{-1}$ recorded the highest plant height and number of leaves due to effective weed control and minimum crop weed competition leading to more nutrient availability resulting in better plant growth attributes.

The Leaf area index differed significantly at all the stages due to the application ofdifferent weed management practices. At harvest, leaf area index of herbicide treatment T5 i.e., Bensulfuron methyl $0.6 \% \mathrm{G}+$ Pretilachlor $6.0 \%$ G 165 g a. i. ha $^{-1} f b$ Bispyribac sodium $10 \%$ SC 10 g a. i. ha ${ }^{-1}$ (T5:3.45), T8 (3.28) and T4 (2.71) were statistically found on par with T12 i.e weed free check (3.77). Whereas, significantly the lowest leaf area index was reported in treatment of un-weeded control i.e., T11 (2.24). Persistence of the assimilatory surface area is pre requisite for a prolonged photosynthetic activity and productivity. Leaf area being the photosynthetic surface plays a vital role in production and availability of photosynthates. Due to less competition from weeds, plots treated with Bensulfuron methyl $0.6 \% \mathrm{G}+$ Pretilachlor $6.0 \%$ G 165 g a. i. ha ${ }^{-1} f b$ Bispyribac sodium $10 \%$ SC 10 g a. i. ha ${ }^{-1}$ recorded higher leaf area as well as leaf area index.

Among the herbicide treatments, significantly higher total dry weight of kodo millet was recorded in treatment received the combination of herbicides Bensulfuron methyl $0.6 \% \mathrm{G}+$ Pretilachlor $6.0 \%$ G $165 \mathrm{~g}$ a. i. $\mathrm{ha}^{-1} \mathrm{fb}$ Bispyribac sodium $10 \%$ SC $10 \mathrm{~g}$ a. i. ha $^{-1}$. The next best herbicide treatment was Bensulfuron methyl $0.6 \% \quad \mathrm{G}+$ Pretilachlor $6.0 \%$ G 165 g a. i. ha ${ }^{-1} f b$ Ethoxysulfuron $15 \%$ WG $12 \mathrm{~g}$ a. i. ha ${ }^{-1}$.This might be attributed to the reduced competition from weeds and increased availability of resources like nutrients, soil moisture and light which paved the way for improvement of crop stature as reflected by taller plants and higher leaf area, which consequently increased the biomass of the crop (Table 1-4). 
Table.1 Category wise weed density (number $\mathrm{m}^{-2}$ ) and weed dry weight $\left(\mathrm{g} \mathrm{m}^{-2}\right)$ at 30 DAS in Kodo millet as influenced by weed management practices

\begin{tabular}{|c|c|c|c|c|c|c|c|c|}
\hline \multirow[t]{2}{*}{ Treatments } & \multicolumn{4}{|c|}{ Weed density } & \multicolumn{4}{|c|}{ Weed dry weight } \\
\hline & Sedge & Grasses & BLWs & Total & Sedge & Grasses & BLWs & Total \\
\hline $\mathbf{T}_{1}$ & $2.37(4.67)$ & $4.79(22.00)$ & $5.00(24.00)$ & $7.18(50.67)$ & $1.47(1.33)$ & $4.42(18.67)$ & $4.57(20.00)$ & $6.40(40.00)$ \\
\hline $\mathbf{T}_{2}$ & $2.69(6.33)$ & $4.14(16.30)$ & $4.15(16.33)$ & $6.31(39.00)$ & $1.95(3.00)$ & $3.70(13.00)$ & $3.57(12.00)$ & $5.36(28.00)$ \\
\hline $\mathbf{T}_{3}$ & 2.77 (6.67) & $4.61(20.33)$ & $4.71(21.33)$ & $7.02(48.33)$ & $2.08(3.33)$ & $4.15(16.33)$ & $4.22(17.00)$ & $6.13(36.67)$ \\
\hline $\mathbf{T}_{4}$ & $1.99(3.00)$ & $4.47(19.30)$ & $3.71(13.33)$ & $6.04(35.67)$ & $1.14(0.33)$ & $4.05(16.00)$ & $3.02(9.00)$ & $5.09(25.33)$ \\
\hline $\mathbf{T}_{5}$ & $2.70(6.33)$ & $2.81(7.00)$ & $2.80(7.00)$ & $4.59(20.33)$ & $1.95(3.00)$ & $2.06(3.33)$ & $1.87(2.66)$ & $3.08(9.00)$ \\
\hline $\mathbf{T}_{6}$ & $2.76(6.67)$ & $3.96(14.70)$ & $3.21(9.33)$ & $5.62(30.67)$ & $2.06(3.33)$ & $3.51(11.33)$ & $2.44(5.00)$ & $4.54(19.67)$ \\
\hline $\mathbf{T}_{7}$ & $2.31(4.33)$ & $4.38(18.70)$ & $4.04(15.33)$ & $6.25(38.33)$ & $1.38(1.00)$ & $3.96(15.33)$ & $3.46(11.00)$ & $5.28(27.33)$ \\
\hline $\mathbf{T}_{8}$ & $2.64(6.00)$ & $2.56(5.70)$ & $2.74(6.66)$ & $4.38(18.33)$ & $1.90(2.67)$ & $1.76(2.33)$ & $1.76(2.33)$ & $2.85(7.33)$ \\
\hline $\mathbf{T}_{9}$ & $2.54(5.67)$ & $4.19(16.70)$ & $3.65(12.33)$ & $5.97(34.67)$ & $1.73(2.33)$ & $3.76(13.33)$ & $3.00(8.00)$ & $4.95(23.67)$ \\
\hline $\mathbf{T}_{10}$ & $1.63(1.67)$ & $1.90(2.70)$ & $2.67(6.33)$ & $3.40(10.67)$ & $1.24(0.67)$ & $1.28(0.67)$ & $1.75(2.66)$ & $2.02(4.00)$ \\
\hline $\mathbf{T}_{11}$ & $3.46(11.00)$ & $5.65(31.000$ & $6.85(46.00)$ & $9.43(88.00)$ & $3.23(9.67)$ & $5.49(29.33)$ & $6.65(43.33)$ & $9.12(82.33)$ \\
\hline $\mathbf{T}_{12}$ & $1.00(0.00)$ & $1.00(0.00)$ & $1.00(0.00)$ & $1.00(0.00)$ & $1.00(0.00)$ & $1.00(0.00)$ & $1.00(0.00)$ & $1.00(0.00)$ \\
\hline S.Em+ & 0.16 & 0.26 & 0.25 & 0.25 & 0.25 & 0.31 & 0.34 & 0.38 \\
\hline C. D. at $5 \%$ & 0.47 & $0.769 .00)$ & 0.76 & 0.75 & 0.74 & 0.92 & 1.00 & 1.12 \\
\hline
\end{tabular}

Data within parentheses are original values; data analyzed using square root $(x+1)$ transformation

DAS-Days after sowing, BLWs= broad leaved weeds. 
Table.2 Category wise weed density (number $\mathrm{m}^{-2}$ ) and weed dry weight $\left(\mathrm{g} \mathrm{m}^{-2}\right)$ at 45DAS in Kodo millet as influenced by Weed Management Practices

\begin{tabular}{|c|c|c|c|c|c|c|c|c|}
\hline \multirow[t]{2}{*}{ Treatments } & \multicolumn{4}{|c|}{ Weed density } & \multicolumn{4}{|c|}{ Weed dry weight } \\
\hline & Sedge & Grasses & BLWs & Total & Sedge & Grasses & BLWs & Total \\
\hline $\mathbf{T}_{1}$ & $2.76(6.67)$ & $4.99(24.00)$ & $5.13(25.33)$ & $7.55(56.00)$ & $1.38(1.00)$ & $4.39(18.33)$ & $4.54(19.66)$ & $6.32(39.00)$ \\
\hline $\mathbf{T}_{2}$ & $2.87(7.33)$ & $4.37(18.30)$ & $4.26(17.33)$ & $6.62(43.00)$ & $1.88(2.67)$ & $3.66(12.67)$ & $3.52(11.66)$ & $5.27(27.00)$ \\
\hline $\mathbf{T}_{3}$ & $2.94(7.67)$ & $4.83(22.30)$ & $4.86(22.66)$ & $7.32(52.66)$ & $1.99(3.00)$ & $4.12(16.00)$ & $4.18(16.66)$ & $6.05(35.67)$ \\
\hline $\mathbf{T}_{4}$ & $2.29(4.33)$ & $4.69(21.30)$ & $3.86(14.66)$ & $6.64(43.33)$ & $1.28(0.67)$ & $4.02(15.67)$ & $2.98(8.66)$ & $5.06(25.00)$ \\
\hline $\mathbf{T}_{5}$ & $2.88(7.33)$ & $3.15(9.00)$ & $2.90(7.66)$ & $4.67(21.00)$ & $1.80(2.33)$ & $1.87(2.67)$ & $1.72(2.00)$ & $2.78(7.00)$ \\
\hline $\mathbf{T}_{6}$ & $2.94(7.67)$ & $4.20(16.70)$ & $3.21(9.33)$ & $5.88(33.66)$ & $1.99(3.00)$ & $2.78(7.00)$ & $2.38(4.66)$ & $3.95(14.67)$ \\
\hline $\mathbf{T}_{7}$ & $2.58(5.67)$ & $4.60(20.70)$ & $4.12(16.00)$ & $6.64(43.33)$ & $1.14(0.33)$ & $3.89(14.67)$ & $3.41(10.66)$ & $5.13(25.67)$ \\
\hline $\mathbf{T}_{8}$ & $2.76(6.67)$ & $2.93(7.70)$ & $3.05(8.66)$ & $4.78(22.00)$ & $1.82(2.33)$ & $1.92(2.83)$ & $1.76(2.33)$ & $2.87(7.50)$ \\
\hline $\mathbf{T}_{9}$ & $2.73(6.67)$ & $4.42(18.70)$ & $3.78(13.33)$ & $6.29(38.66)$ & $1.62(2.00)$ & $3.72(13.00)$ & $2.94(7.66)$ & $4.85(22.67)$ \\
\hline $\mathbf{T}_{10}$ & $1.82(2.33)$ & $2.14(3.70)$ & $2.85(7.33)$ & $3.78(13.33)$ & $1.14(0.33)$ & $1.21(0.50)$ & $1.69(2.33)$ & $1.86(3.17)$ \\
\hline $\mathbf{T}_{11}$ & $3.20(9.33)$ & $5.47(29.00)$ & $6.72(44.33)$ & $9.14(82.66)$ & $3.40(10.67)$ & $5.61(30.67)$ & $6.75(44.66)$ & $9.32(86.00)$ \\
\hline $\mathbf{T}_{12}$ & $1.00(0.00)$ & $1.00(0.00)$ & $1.00(0.00)$ & $1.00(0.00)$ & $1.00(0.00)$ & $1.00(0.00)$ & $1.00(0.00)$ & $1.00(0.00)$ \\
\hline S.Em+ & 0.16 & 0.24 & 0.30 & 0.23 & 0.20 & 0.32 & 0.31 & 0.34 \\
\hline C. D. at $5 \%$ & 0.47 & 0.71 & 0.88 & 0.70 & 0.61 & 0.95 & 0.91 & 1.01 \\
\hline
\end{tabular}

Data within parentheses are original values; data analyzed using square root $(x+1)$ transformation DAS-Days after sowing, BLWs= broad leaved weeds 
Table.3 Category wise weed density (number $\mathrm{m}^{-2}$ ) and weed dry weight $\left(\mathrm{g} \mathrm{m}^{-2}\right)$ at harvest in Kodo millet as influenced by Weed Management Practices

\begin{tabular}{|c|c|c|c|c|c|c|c|c|}
\hline \multirow[t]{2}{*}{ Treatments } & \multicolumn{4}{|c|}{ Weed density } & \multicolumn{4}{|c|}{ Weed dry weight } \\
\hline & Sedge & Grasses & BLWs & Total & Sedge & Grasses & BLWs & Total \\
\hline $\mathbf{T}_{1}$ & $2.23(4.66)$ & $5.61(30.66)$ & $3.95(14.66)$ & $7.13(50.00)$ & $1.71(1.93)$ & $2.65(6.03)$ & $3.40(10.66)$ & $4.42(18.63)$ \\
\hline $\mathbf{T}_{2}$ & $2.35(4.00)$ & $5.21(26.33)$ & $3.82(13.66)$ & $6.69(44.00)$ & $1.78(2.22)$ & $2.61(5.82)$ & $3.31(10.00)$ & $4.36(18.04)$ \\
\hline $\mathbf{T}_{3}$ & $2.18(3.33)$ & $5.35(27.66)$ & $4.35(18.00)$ & $7.06(49.00)$ & $1.75(2.06)$ & $2.49(5.21)$ & $3.21(9.33)$ & $4.20(16.60)$ \\
\hline $\mathbf{T}_{4}$ & $1.63(5.33)$ & $5.02(24.33)$ & $3.74(13.00)$ & $6.60(42.66)$ & $1.39(1.02)$ & $2.23(4.07)$ & $3.16(9.00)$ & $3.88(14.09)$ \\
\hline $\mathbf{T}_{5}$ & $2.48(65.15)$ & $3.81(13.66)$ & $2.55(5.66)$ & $4.69(21.00)$ & $1.49(1.21)$ & $1.33(0.83)$ & $1.63(1.66)$ & $2.17(3.71)$ \\
\hline $\mathbf{T}_{6}$ & $2.14(3.66)$ & $5.08(25.00)$ & $4.00(15.00)$ & $6.68(43.66)$ & $1.52(1.32)$ & $2.39(4.78)$ & $2.27(4.33)$ & $3.38(10.43)$ \\
\hline $\mathbf{T}_{7}$ & $1.72(3.00)$ & $4.12(23.66)$ & $3.87(14.00)$ & $6.45(40.66)$ & $1.56(1.45)$ & $2.33(4.58)$ & $2.50(5.33)$ & $3.49(11.36)$ \\
\hline $\mathbf{T}_{8}$ & $1.99(2.00)$ & $3.40(10.66)$ & $2.87(8.66)$ & $4.72(21.33)$ & $1.68(1.87)$ & $1.54(1.4)$ & $2.43(5.00)$ & $3.04(8.27)$ \\
\hline $\mathbf{T}_{9}$ & $2.30(4.33)$ & $4.89(23.00)$ & $3.81(13.66)$ & $6.47(41.00)$ & $1.66(1.77)$ & 2.17 & $2.81(7.00)$ & $3.67(12.64)$ \\
\hline $\mathbf{T}_{10}$ & $1.28(0.66)$ & $3.31(10.00)$ & $1.91(2.66)$ & $3.78(13.33)$ & $1.20(0.46)$ & $1.38(1.00)$ & $1.36(0.85)$ & $1.81(2.32)$ \\
\hline$T_{11}$ & $2.99(8.00)$ & $6.25(38.33)$ & $6.95(47.33)$ & $9.72(93.66)$ & $3.64(12.33)$ & $5.64(31.00)$ & $6.24(38.00)$ & $8.00(63.00)$ \\
\hline $\mathbf{T}_{12}$ & $1.00(0.00)$ & $1.00(0.00)$ & $1.00(0.00)$ & 1.00 & $1.00(0.00)$ & $1.00(0.00)$ & $1.00(0.00)$ & $1.00(0.00)$ \\
\hline S.Em+ & 0.18 & 0.27 & 0.13 & 0.21 & 0.11 & 0.20 & 0.19 & 0.20 \\
\hline C. D. at $5 \%$ & 0.53 & 0.79 & 0.39 & 0.64 & 0.35 & 0.60 & 0.57 & 0.58 \\
\hline
\end{tabular}

Data within parentheses are original values; data analyzed using square root $(x+1)$ transformation DAS-Days after sowing, BLWs= Broad leaved weeds 
Table.4 Different growth parameters, weed control efficiency and phytotoxicity of kodo millet as influenced by weed management practices

\begin{tabular}{|c|l|c|c|c|c|c|}
\hline Treatments & $\begin{array}{c}\text { Plant height } \\
(\mathbf{c m})\end{array}$ & $\begin{array}{c}\text { LAIat } \\
\text { harvest }\end{array}$ & $\begin{array}{c}\text { Total dry weight } \\
\left(\mathbf{g}_{\mathbf{~ p l a n t}}^{\mathbf{1}} \mathbf{)}\right.\end{array}$ & $\begin{array}{c}\text { WCE at 45 } \\
\text { DAS }\end{array}$ & $\begin{array}{c}\text { WCE at } \\
\text { harvest }\end{array}$ & $\begin{array}{c}\text { Phytotoxicity } \\
\text { score }\end{array}$ \\
\hline $\mathbf{T}_{\mathbf{1}}$ & 57.01 & 2.37 & 43.30 & 54.6 & 70.42 & 2 \\
\hline $\mathbf{T}_{\mathbf{2}}$ & 57.67 & 2.64 & 50.10 & 68.6 & 71.36 & 0 \\
\hline $\mathbf{T}_{\mathbf{3}}$ & 57.33 & 2.56 & 47.53 & 58.5 & 73.65 & 0 \\
\hline $\mathbf{T}_{\mathbf{4}}$ & 68.19 & 2.71 & 55.33 & 70.9 & 75.98 & 2 \\
\hline $\mathbf{T}_{\mathbf{5}}$ & 69.62 & 3.45 & 65.20 & 91.8 & 94.63 & 0 \\
\hline $\mathbf{T}_{\mathbf{6}}$ & 68.33 & 2.96 & 61.70 & 82.9 & 83.44 & 0 \\
\hline $\mathbf{T}_{\mathbf{7}}$ & 57.33 & 2.76 & 52.40 & 70.1 & 81.96 & 2 \\
\hline $\mathbf{T}_{\mathbf{8}}$ & 68.31 & 3.28 & 62.67 & 91.2 & 86.87 & 0 \\
\hline $\mathbf{T}_{\mathbf{9}}$ & 57.28 & 2.82 & 59.00 & 73.6 & 79.93 & 0 \\
\hline $\mathbf{T}_{\mathbf{1 0}}$ & 70.31 & 3.37 & 65.20 & 96.3 & 96.31 & - \\
\hline $\mathbf{T}_{\mathbf{1 1}}$ & 55.39 & 2.24 & 28.17 & 100 & 100 & - \\
\hline $\mathbf{T}_{\mathbf{1 2}}$ & 70.44 & 3.77 & 66.83 & 0.00 & 0.00 & - \\
\hline S.Em+ & 3.70 & 0.24 & 2.17 & 54.6 & 70.42 & - \\
\hline C. D. at 5\% & 10.85 & 0.71 & 6.36 & 68.6 & 71.36 & - \\
\hline
\end{tabular}

$\mathrm{T}_{1}$ : Oxadiargyl 80 WP @ $150 \mathrm{~g}$ a. i. ha ${ }^{-1}, \mathrm{~T}_{2}$ :Bensulfuron methyl $0.6 \mathrm{G}+$ Pretilachlor $6.0 \mathrm{G}\left(165 \mathrm{~g} \mathrm{a}\right.$ i/ha), $\mathrm{T}_{3}:$ Pendimethalin $38.7 \mathrm{CS}$ (450 g a. i/ha), $\mathrm{T}_{4}$ :

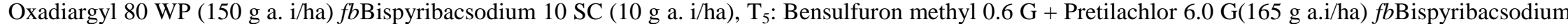
$10 \mathrm{SC}\left(10 \mathrm{~g}\right.$ a. i/ha), $\mathrm{T}_{6}$ : Pendimethalin $38.7 \mathrm{CS}$ (450 g a. i/ha) fbBispyribac sodium $10 \mathrm{SC}$ (10 g a.i/ha), $\mathrm{T}_{7}$ : Oxadiargyl $80 \mathrm{WP}$ (150 g a.i/ha) $f b$ Ethoxysulfuron 15 WG (12 g a.i/ha), $\mathrm{T}_{8}$ : Bensulfuron methyl $0.6 \mathrm{G}+$ Pretilachlor $6.0 \mathrm{G}(165 \mathrm{~g} \mathrm{a}$. i/ha) fbEthoxysulfuron $15 \mathrm{WG}$ (12 g a. i/ha), Tg: Pendimethalin $38.7 \mathrm{CS}$ (450 $\mathrm{g}$ a. i/ha) $f b$ Ethoxysulfuron $15 \mathrm{WG}$ (12 g a.i/ha), $\mathrm{T}_{10}$ : Two intercultivation + hand weeding @ 20 \& 40 DAS, $\mathrm{T}_{11}$ : Un weeded check, $\mathrm{T}_{12}$ : Weed free check. DAS-Days after sowing 
Whereas, the lower total plant dry weight was recorded in un-weeded control $\left(\mathrm{T}_{11}\right)$, as a result of severe weed interference and constant competition throughout the crop growth period which suppressed the growth of kodo millet crop, as projected by the studies of Arunachalam et al., (1995) and Travis and James (2016).

Highest weed control efficiency in weed free check $\left(T_{12}\right)$ was due to highest efficiency of human labour in removing all the types of weeds. Bensulfuron methyl $0.6 \% \mathrm{G}+$ Pretilachlor $6.0 \%$ G 165 g a. i. ha ${ }^{-1} f b$ Bispyribac sodium $10 \%$ SC $10 \mathrm{~g}$ a. i. $\mathrm{ha}^{-1}$ recorded next higher weed control efficiency due to control of broad spectrum of weeds including sedge, grasses and broad leaf weeds.

Whereas the lowest weed control efficiency in $\mathrm{T}_{4}$ i.e., Oxadiargyl $80 \%$ WP $150 \mathrm{~g}$ a. i. $\mathrm{ha}^{-1} \mathrm{fb}$ Bispyribac sodium $10 \%$ SC $10 \mathrm{~g}$ a. i. ha $^{-1}$ was due to control of only some specific weed flora like broad leaf weeds more efficiently, sedge to some extent and no control of grassy weeds. These results are in accordance with Saha (2009) and Singh et al., (2005).

In conclusion the application of Bensulfuron methyl $0.6 \% \mathrm{G}+$ Pretilachlor $6.0 \%$ G $165 \mathrm{~g}$ a. i. $\mathrm{ha}^{-1} \mathrm{fb}$ Bispyribac sodium $10 \%$ SC $10 \mathrm{~g}$ a. i. ha $^{-1}$ is more effective due to control of the broad spectrum of weeds more efficiently and thereby increasing all the growth parameters of kodo millet.

\section{References}

Arunchalam, A. A., Veerabadran, V. and Muthusankara N, A., 1995, Integrated nitrogen supply system for finger millet. Indian J. Agron., 40: 109-111.

Pradhan, A. S., Rajput. and Thakur, A., 2010, Effect of weed management on growth and yield of finger millet. Indian $J$. Weed Sci., 42(2): 53-56.

Saha, S., 2009, Efficacy of bensulfuronmethyl for controlling sedges and non grassy weeds in transplanted rice (Oryza sativa L.). Indian J. Agric. Sci., 75(1): 140-143.

Singh, G., Singh and Mahendra Singh, 2005, Effect of bensulfuron-methyl on sedges and weeds in transplanted rice. Indian J. Weed Sci., 48 (3\&4): 87-91., V. P.,

Travis, W. J. and James, G., W., 2016, Weed control in corn (Zea mays L.) as influenced by pre and post emergence herbicides. Int. J. Agron., Volume 2016, Article ID 2607671, 9 pages.

Yaduraju, N. T., 2006, Herbicide resistant crop in weed management in The extended summaries, Golden Jubliee National Symposium on Conservation Agriculture and Environment. Banaras Hindu University, Varanasi.,pp: 297298.

\section{How to cite this article:}

Meghana, G. K., T. S. Sukanya, R. M. Salmankhan and Kiran, H. P. 2020. Effect of Weed Management Practices on Weed Density, Dry Weight and Growth of Kodo Millet (Paspalum scrobiculatum L.). Int.J.Curr.Microbiol.App.Sci. 9(11): 3377-3384.

doi: https://doi.org/10.20546/ijcmas.2020.911.403 